\title{
Doenças da pele relacionadas à poluição do ar: uma revisão sistemática
}

\section{Skin diseases related to air pollution: a systematic review}

\author{
José Otávio Batista Leite¹, Boscolli Barbosa Pereira² (orcid. org/0000-0002-2633-9067)
}

1. Centro de Referência Nacional em Dermatologia Sanitária e Hanseníase da Universidade Federal de Uberlândia (UFU), Uberlândia, MG, Brasil. 2. Docente do Curso de Saúde Ambiental da Universidade Federal de Uberlândia (UFU), Uberlândia, MG, Brasil.

\section{Resumo}

Introdução: A poluição atmosférica compreende uma mistura complexa de diversos contaminantes particulados (fumaça de cigarro, gases de combustão veicular e compostos orgânicos voláteis, entre outros). Recentemente, algumas investigações revelaram a existência de associação direta entre a poluição atmosférica e as doenças de pele. Objetivos: Investigar e discutir aspectos relevantes no cenário da pesquisa epidemiológica que trata da relação entre a poluição atmosférica e as doenças de pele em humanos. Métodos: Foi realizada uma revisão sistemática da literatura científica nacional e internacional, que avaliou os artigos publicados na última década, que trataram de doenças de pele em humanos relacionadas à poluição atmosférica. Resultados: A presente revisão identificou 288 estudos que abordaram a temática, dos quais 34 atenderam aos critérios de inclusão previamente estabelecidos. Os resultados revelam que a exposição à fumaça de cigarro e às emissões veiculares está altamente associada com o desenvolvimento de várias doenças de pele, sendo o estresse oxidativo, com a participação de citocinas, a principal via inflamatória. Conclusão: Conclui-se que o controle da exposição ambiental é a principal forma de prevenção; e o tratamento das doenças de pele associadas à poluição atmosférica pode ser realizado por meio da utilização de drogas anti-inflamatórias e antioxidantes.

Palavras-chave: Poluentes atmosféricos. Estresse oxidativo. Doenças inflamatórias. Câncer de pele. Alopécia.

\begin{abstract}
Introduction: Atmospheric pollution contains a complex mixture of various particulate contaminants (cigarette smoke, vehicular emissions, volatile organic compounds). Recently, several investigations have revealed the existence of a direct association between air pollution and skin diseases. Objectives: To investigate and discuss relevant aspects of the epidemiological research concern the relationship between air pollution and skin diseases in humans. Methods: A systematic review of national and international scientific literature was conducted, evaluating the articles published in the last decade, which tried to investigate skin diseases in humans in relation to air pollution. Results: This review identified 288 studies that addressed the theme of which 34 met the inclusion criteria previously established. Results show that exposure to cigarette smoke and vehicle emissions is highly associated with the development of various skin diseases, and oxidative stress, with the participation of cytokines is the main inflammatory pathway. Conclusion: environmental exposure control is the primary means of prevention and treatment of skin diseases associated to air pollution, something that can be accomplished through the use of anti-inflammatory and anti-oxidants drugs.
\end{abstract}

Key words: Benefits. Autism. Physical activity.

\section{INTRODUÇÃO}

Os efeitos do ambiente sobre a pele humana são conhecidos por provocarem uma série de alterações estruturais que podem causar desfechos como envelhecimento cutâneo ${ }^{1}$, doenças inflamatórias ${ }^{2}$ e câncer ${ }^{3}$. Ainda que a radiação ultravioleta (UV) seja a principal causa dessas alterações, a poluição atmosférica tem sido reconhecida por contribuir para a ocorrência de doenças de pele ${ }^{4}$.

Os poluentes presentes no ar incluem várias substâncias que podem produzir efeitos deletérios na saúde humana. Em ambientes externos, os principais poluentes atmosféricos são o monóxido de carbono (CO); os hidrocarbonetos ( $\mathrm{HC})$; os materiais particulados (MP); os óxidos de nitrogênio (NOx) e os óxidos de enxofre $(\mathrm{SOx})^{5}$. Esses poluentes têm origem no processo de combustão dos combustíveis fósseis utilizados nos veículos, causando a liberação dessas substâncias químicas, que tendem a se concentrar na atmosfera, sofrendo, inclusive, modificações, como nas reações fotoquímicas, que formam o ozônio $(\mathrm{O} 3)^{6}$.

Em ambientes fechados, os principais poluentes relacionados aos problemas de saúde em humanos são aqueles provenientes da fumaça do cigarro e os utilizados em produtos domissanitários, tintas e solventes, incluindo compostos orgânicos voláteis (COVs), como benzeno, tolueno, etilbenzeno, xileno e outros ${ }^{7}$. Embora não existam evidências de que os poluentes possam entrar no organismo através dos poros presentes na pele, não há dúvidas, segundo vários relatos publicados na literatura 
científica, de que partículas potencialmente tóxicas podem penetrar a pele, passando pelos folículos pilosos. Considerando que os folículos pilosos presentes no escalpo e face, que estão em constante contato com a atmosfera, representam aproximadamente $10 \%$ da superfície total da pele, é evidente a importância da via transfolicular de entrada de poluentes ${ }^{8}$.

Nesse sentido, os estudos em Dermatotoxicologia têm ganhado importância na literatura científica internacional, na medida em que reconhecem a pele como órgão-alvo e via de entrada para compostos com potencial tóxico, quer sejam aqueles presentes nos dermocosméticos e medicamentos, quer sejam aqueles que são transportados pelo ar.

Com base no exposto e, em razão da crescente preocupação com a saúde da pele e a necessidade de subsidiar reflexões e ações que contribuam para o desenvolvimento da Dermatotoxicologia no Brasil, o presente estudo tem como objetivo principal contribuir com informações atualizadas aos profissionais de saúde no que se refere ao diagnóstico, à prevenção e ao tratamento das doenças de pele associadas aos poluentes atmosféricos.

Para alcançar o objetivo proposto, foi realizada uma revisão sistemática de artigos da literatura científica nacional e internacional, publicados nos últimos dez anos, os quais descrevem resultados de pesquisas clínica, experimental ou epidemiológica e tratam da associação entre poluentes do ar e doenças de pele.

Buscou-se, por meio dessa revisão, a obtenção de informações atualizadas sobre as principais doenças de pele associadas à poluição atmosférica; os tipos de poluentes relacionados aos desfechos avaliados; os grupos de pessoas investigados e o período e o local de realização dos estudos.

\section{METODOLOGIA}

No presente trabalho, foi realizada uma revisão sistemática dos artigos publicados em periódicos indexados, nacional e internacionalmente, que abordaram, por meio de pesquisa clínica, experimental ou epidemiológica, a associação entre poluição atmosférica e doenças de pele em humanos.

Para realização da revisão sistemática, foram incluídos somente artigos originais publicados entre agosto de 2006 e julho de 2016, em língua portuguesa ou inglesa, resultantes de investigações realizadas no Brasil, ou em quaisquer outros países, e que apresentaram clareza quanto aos seguintes critérios de inclusão: (i) informações sobre a doença de pele associada à poluição atmosférica; (ii) informação sobre o ano/ período de realização da pesquisa; (iii) identificação do tipo de poluente atmosférico associado à ocorrência da doença de pele em humanos; (iv) informações sobre os grupos de pessoas estudadas (crianças, adolescentes, adultos ou idosos); (v) informação sobre o país onde o estudo foi realizado.
No processo de seleção dos artigos para a realização da revisão sistemática, não houve restrição com relação ao sexo da população estudada, nem quanto ao tempo de exposição. Contudo, não foram considerados os artigos de revisão, metanálises, editoriais e os artigos que tratavam de pesquisas realizadas com a utilização de modelos experimentais animais.

O processo de levantamento dos artigos, segundo os critérios de inclusão estabelecidos, foi realizado nos bancos de dados Literatura Latino-Americana e do Caribe em Ciências da Saúde (LILACS), Medical Literature Analysis and Retrieval System Online (MEDLINE/PUBMED), Web of Science, ScienceDirect, Scopus, Scientific Electronic Library Online (SciELO) e COCHRANE. A busca integrada nos campos título, resumo e assunto, disponíveis nos bancos de dados, foi realizada pela combinação dos seguintes descritores: "POLUIÇÃO ATMOSFÉRICA" ou "ATMOSPHERIC POLLUTION"; "POLUIÇÃO DO AR" ou "AIR POLLUTION"; "DOENÇAS DE PELE" ou "SKIN DISEASES".

Os artigos selecionados, de acordo com os critérios estabelecidos, foram analisados na íntegra. A ferramenta PRISMA (preferred reporting items for systematic reviews and meta-analyses) foi utilizada para orientar a redação da revisão sistemática9 ${ }^{9}$.

\section{RESULTADOS E DISCUSSÃO}

O processo de busca e de seleção dos artigos que compõem a presente revisão está representado na Figura 1.

Figura 1. Diagrama da seleção de artigos para revisão sistemática
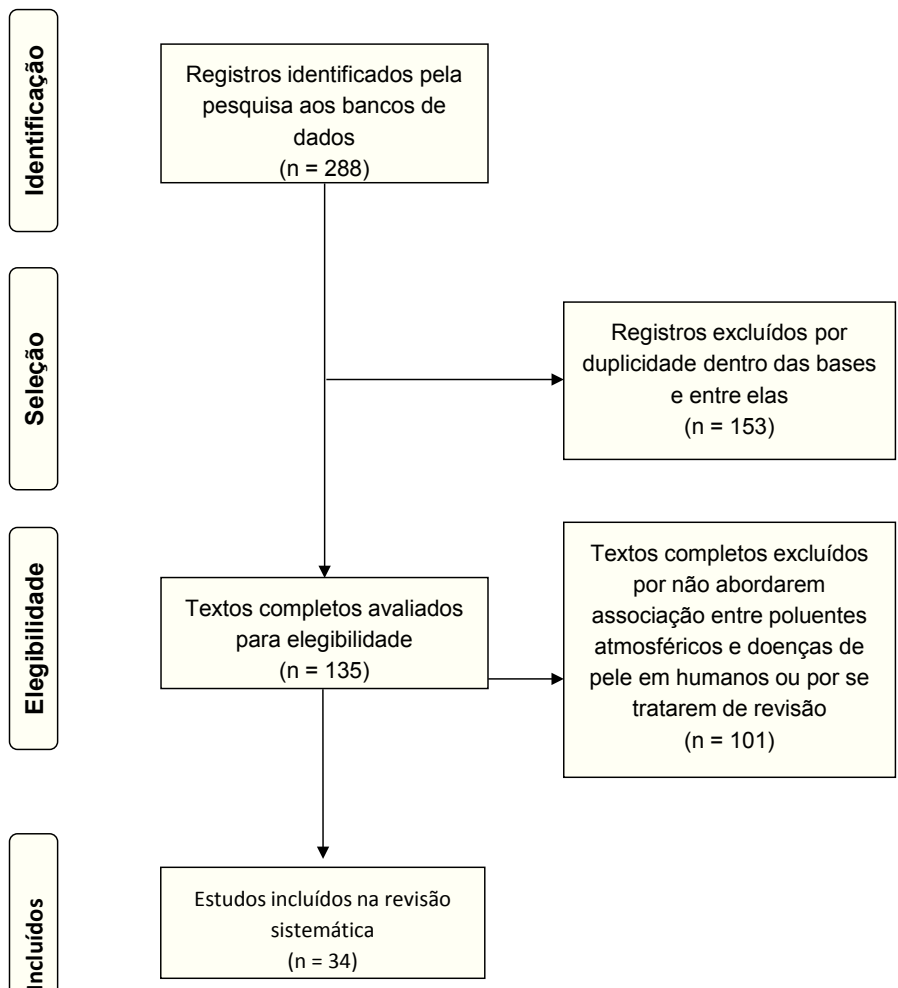
Após a exclusão dos artigos em duplicidade, ficaram elegíveis 135 estudos, dos quais 34, por atenderem aos critérios prédefinidos, foram incluídos na revisão sistemática. Informações sobre o tipo de doença de pele associada, ano/período de realização do estudo, poluentes atmosféricos associados à doença investigada, grupo populacional estudado e país onde foi realizado o estudo são apresentadas na Tabela 1.

Tabela 1. Caracterização dos estudos quanto à doença avaliada, ao ano/período de realização do estudo, aos poluentes associados ao desfecho, ao grupo populacional estudado e ao local de realização do estudo.

\begin{tabular}{|c|c|c|c|c|c|}
\hline Referências & Doença avaliada & Ano/período do estudo & $\begin{array}{l}\text { Poluentes associados } \\
\text { positivamente }\end{array}$ & População estudada & País de estudo \\
\hline $\mathrm{Li}$, et al1 & $\begin{array}{l}\text { Envelhecimento } \\
\text { cutâneo }\end{array}$ & $2012 ; 2013$ & Covs & Adultos e idosos & China \\
\hline Brans, et al2 & Dermatite atópica & $2005-2009$ & Fumaça de cigarro & Adultos & Alemanha \\
\hline Fazzo, et al3 & Câncer de pele & 1999-2006 & Asbesto & Adultos & Itália \\
\hline Pénard-Morand, et al7 & Dermatite atópica & $1999 ; 2000$ & $\mathrm{MP}_{10} ; \mathrm{CO} ; \mathrm{NO}_{\mathrm{x}} ;$ Benzeno & Crianças & França \\
\hline Kwon et $\mathrm{al}^{10}$ & Dermatite atópica & $2006-2012$ & COVs & Crianças & Coreia do Sul \\
\hline Lee, et $\mathrm{al}^{11}$ & Dermatite atópica & $1995-2001$ & Fumaça de cigarro & Adolescentes & Taiwan \\
\hline Kallawicha, et al ${ }^{12}$ & Dermatite atópica & $2011 ; 2012$ & Bioaerossol & Adultos & Taiwan \\
\hline Solé et $\mathrm{a}^{13}$ & Dermatite atópica & 2006 & $\mathrm{SO}_{2}$ & Adolescentes & Brasil \\
\hline Gatherwright, et al ${ }^{15}$ & Alopécia & $2009-2011$ & Fumaça de cigarro & Adultos & Estados Unidos \\
\hline Gatherwright, et $\mathrm{al}^{16}$ & Alopécia & $2009-2011$ & Fumaça de cigarro & Adultos & Estados Unidos \\
\hline Kim et $\mathrm{al}^{17}$ & Dermatite atópica & 2009 & $\mathrm{MP}_{10} ; \mathrm{CO}_{2}$ & Crianças & Coreia do Sul \\
\hline Kim et $\mathrm{al}^{18}$ & Dermatite atópica & $2009-2010$ & $\mathrm{MP}_{10} ; \mathrm{MP}_{2,5} ;$ Tolueno & Crianças & Coreia do Sul \\
\hline Kathuria; Silverberg ${ }^{19}$ & Dermatite atópica & $2006 ; 2007$ & $\begin{array}{l}\mathrm{MP}_{10} ; \mathrm{MP}_{2,5} ; \mathrm{CO} ; \mathrm{NO}_{3} ; \\
\mathrm{NO}_{2} ; \mathrm{SO}_{3} ; \mathrm{SO}_{2} ; \text { Arsênio; } \\
\text { Níquel; Chumbo; } \\
\text { Vanádio; Cádmio; Cobre } \\
\text { e Potássio }\end{array}$ & Crianças & Estados Unidos \\
\hline Su; Chen ${ }^{20}$ & Alopécia & 2005 & Fumaça de cigarro & Adultos e idosos & Coreia do Sul e Taiwan \\
\hline Vierkötter, et al ${ }^{21}$ & $\begin{array}{l}\text { Envelhecimento } \\
\text { cutâneo }\end{array}$ & $2008 ; 2009$ & $\mathrm{MP}_{10}$ & Idosos & Alemanha \\
\hline Altuğ, et $\mathrm{al}^{24}$ & Dermatite atópica & 2008; 2009 & $\mathrm{O}_{3} ; \mathrm{NO}_{2} ; \mathrm{SO}_{2}$ & Crianças & Turquia \\
\hline Graif, et al ${ }^{25}$ & Dermatite atópica & 1998 & Fumaça de cigarro & Adolescentes & Israel \\
\hline Herbarth, et $\mathrm{al}^{26}$ & Dermatite atópica & 1998 & COVs & Crianças & Alemanha \\
\hline Huang, et $\mathrm{al}^{27}$ & Dermatite atópica & 2005 & $\mathrm{CO}$ & Crianças & Taiwan \\
\hline Jedrychowski et al ${ }^{28}$ & Dermatite atópica & 2001-2004 & $\mathrm{MP}_{2,5}$ & Crianças & Polônia; Estados Unidos \\
\hline Kim, et al ${ }^{29}$ & Dermatite atópica & 2009; 2010 & Benzeno; Tolueno & Crianças & Coreia do Sul \\
\hline Krämer, et al $\left.\right|^{30}$ & Dermatite atópica & 1995-1999 & $\mathrm{NO}_{2}$ & Crianças & Alemanha \\
\hline Lee, et $\mathrm{al}^{31}$ & Dermatite atópica & 1994 & $\mathrm{MP}_{10} ; \mathrm{CO} ; \mathrm{NO}_{x^{\prime}} ; \mathrm{SO}_{2}$ & Crianças & Taiwan \\
\hline Lee, et $\mathrm{al}^{32}$ & Dermatite atópica & 2008 & Fumaça de cigarro & Adultos & Taiwan \\
\hline Lee, et $\mathrm{al}^{33}$ & Dermatite atópica & $2008-2010$ & COVs & Crianças & Coreia do Sul \\
\hline Lee, et $\mathrm{a}^{34}$ & Dermatite atópica & $2008 ; 2010$ & $\mathrm{MP}_{10} ; \mathrm{CO} ; \mathrm{CO}_{2} ; \mathrm{NO}_{2}$ & Crianças & Coreia do Sul \\
\hline Morgenstern, et $\mathrm{al}^{35}$ & Dermatite atópica & 1999; 2000 & $\mathrm{MP}_{2,5} ; \mathrm{NO}_{2}$ & Crianças & Alemanha \\
\hline $\begin{array}{l}\text { Schmitz; Atzpodien; } \\
\text { Schlaud }^{36}\end{array}$ & Dermatite atópica & $2003-2006$ & Fumaça de cigarro & Crianças e adolescentes & Alemanha \\
\hline Seo, et $\mathrm{al}^{37}$ & Dermatite atópica & 2011 & $\mathrm{MP}_{10}$ & Adultos & Coreia do Sul \\
\hline Song et $\mathrm{al}_{38}$ & Dermatite atópica & 2009 & $\mathrm{MP}_{10} ; \mathrm{MP}_{2,5} ; \mathrm{MP}_{1,0}$ & Crianças & Coreia do Sul \\
\hline Wang et $\mathrm{al}^{39}$ & Dermatite atópica & 2004 & Fumaça de cigarro & Crianças & Taiwan \\
\hline Wang et $\mathrm{al}^{40}$ & Dermatite atópica & 2007 & Fumaça de cigarro & Crianças & Taiwan \\
\hline Wang et $\mathrm{al}^{41}$ & Dermatite atópica & 2008 & Fumaça de cigarro & Crianças & Taiwan \\
\hline Yi et al42 & Dermatite atópica & $2005-2007$ & Fumaça de cigarro & Crianças & Coreia do Sul \\
\hline
\end{tabular}


Quanto às doenças de pele relacionadas à exposição aos poluentes atmosféricos, a maioria $(82,3 \%)$ dos estudos avaliou a ocorrência de dermatite atópica. Os desfechos alopécia, envelhecimento cutâneo e câncer de pele apresentaram representatividade de $8,9 \% ; 5,9 \%$ e $2,9 \%$ dos trabalhos avaliados, respectivamente.

Ainda que a presente revisão tenha avaliado trabalhos publicados nos últimos dez anos, 44,1\% destes estudos analisados foram iniciados ou completamente realizados em períodos anteriores a 2006. Esse resultado aponta para duas evidências. A primeira delas diz respeito ao atraso nas publicações, como ocorre no trabalho de Fazzo, et al ${ }^{3}$, que foi publicado após dez anos da realização do estudo. A segunda evidência consiste na realização de estudos de longa duração, como estudos de coorte, realizados em 35,3\% dos trabalhos avaliados nessa revisão. Kwon et al., $(2015)^{10}$ e Lee et al., $(2007)^{11}$, por exemplo, realizaram estudos que tiveram duração de sete anos.

Com relação aos poluentes atmosféricos associados às doenças de pele investigadas, em todos os estudos, foi observada associação estatisticamente significativa entre as concentrações dos poluentes monitorados e as doenças de pele avaliadas. Também foi observado que a maior parte dos estudos revisados $(55,8 \%)$ abordou poluentes oriundos das emissões veiculares e industriais, sendo que 10 dos 34 trabalhos encontraram associação positiva entre a exposição ao material particulado (MP) e a ocorrência de dermatite atópica. Em menor proporção, a associação entre doenças de pele e fumaça de cigarro foi relatada em $32,3 \%$ dos trabalhos avaliados. Associações entre doenças de pele e outros poluentes foram pesquisadas nos trabalhos de Fazzo et al.3 e Kallawicha, et al $^{12}$, que investigaram a exposição ao asbesto e bioaerossol, respectivamente.

Sobre os grupos populacionais avaliados, verificou-se que as crianças $(58,8 \%)$ foram mais frequentemente investigadas e confirmadas como o segmento mais susceptível aos efeitos da poluição atmosférica. Em adultos, os efeitos do ambiente contaminado nas doenças de pele foram investigados quanto ao contexto da exposição ocupacional e à fumaça do cigarro.

Quanto ao local de realização dos estudos, houve predomínio de trabalhos realizados nos continentes asiático e europeu, com representatividade de $52,9 \%$ e $29,4 \%$, respectivamente. No Brasil, apenas o trabalho de Solé, et $a^{13}$, que abordou a prevalência de dermatite atópica em adolescentes expostos a poluentes provenientes do tráfego veicular em grandes centros urbanos, esteve presente na revisão. Esse cenário indica a necessidade (e possibilidade) de crescimento de estudos dermatotoxicológicos no Brasil, tanto nos campos da prevenção e do diagnóstico, quanto no que se refere aos tratamentos para as doenças de pele.

A poluição atmosférica, resultante dos processos de industrialização e urbanização, tem como principal agente causador de efeitos danosos à saúde o material particulado ${ }^{14}$, que compreende, além de fuligem e alérgenos de ácaros e pólen, contaminantes gasosos, como sulfatos $\left(\mathrm{SO}_{x}, \mathrm{SO}_{2}\right.$ e $\left.\mathrm{SO}_{3}\right)$, nitratos (NOX, NO2 e NO3) e compostos derivados do carbono $\left(\mathrm{CO}\right.$ e $\left.\mathrm{CO}_{2}\right)$.

A presente revisão evidenciou a associação do material particulado (e contaminantes relacionados) à ocorrência de doenças de pele oriundas de processos inflamatórios, como as dermatites atópicas. As exposições de natureza crônica ao material particulado, especialmente ao $\mathrm{MP}_{2,5}$, que tem os veículos como principal fonte de emissão, constituem o fator ambiental mais influente na prevalência das dermatites ${ }^{18,} 19,28$, 35, 38. Na mesma direção, outro achado importante consiste na elevada frequência de trabalhos que mostraram associação entre a exposição à fumaça de cigarro e o diagnóstico de dermatites atópicas e alopécia, tanto em homens quanto em mulheres.

Em todos esses trabalhos, a principal hipótese defendida é a de que os poluentes presentes no material particulado e na fumaça do cigarro induzem processos de estresse oxidativo na pele, causando disfunções na barreira cutânea ou desregulação imunológica, como esclarecem os trabalhos de Gatherwright, et $\mathrm{al}^{15,16}, \mathrm{Kim}$, et $\mathrm{al}^{17,18}$ e Kathuria e Silverberg ${ }^{19}$. De forma mais específica, esses trabalhos destacam a formação de espécies reativas de oxigênio (ROS) e a produção de citocinas próinflamatórias como resultado da exposição aos poluentes atmosféricos presentes no material particulado e na fumaça do cigarro. $O$ aumento das espécies reativas de oxigênio está diretamente associado à diminuição da produção de colágeno e, consequentemente, da principal função da pele, que é prevenir a entrada de patógenos. Além disso, esse processo de estresse oxidativo também é danoso aos mecanismos de reparo de DNA $^{1}$. Assim, esses fatores, associados, resultam nos processos inflamatórios e no envelhecimento do tecido epitelial ${ }^{21 .}$

É importante ressaltar que, embora a dermatite atópica tenha sido associada à exposição aos poluentes provenientes do material particulado ou da fumaça de cigarro, os trabalhos de Gatherwright, et $\mathrm{al}^{15,16}$ e de Su e Chen ${ }^{20}$, que estudaram a ocorrência de alopécia em adultos e idosos, relacionaram esse desfecho ao contato com a fumaça de cigarro estritamente.

Entre os trabalhos avaliados na presente revisão, apenas o estudo de Fazzo, et $\mathrm{al}^{3}$ abordou a relação entre poluente atmosférico e câncer de pele, sugerindo a associação de asbesto, um conhecido agente carcinogênico, com a ocorrência de melanoma em indivíduos expostos a uma região contaminada por resíduos industriais na Itália. Contudo, diversos estudos epidemiológicos evidenciam que a exposição aos poluentes atmosféricos constitui fator de risco elevado para a ocorrência de câncer de pele, especialmente, do tipo melanoma. A exposição aos contaminantes carreados pelo material particulado, por exemplo, induz alterações celulares no epitélio, como a formação de actina $F$, e aumenta a expressão de proteínas reguladoras do ciclo celular, como a proteína citoplasmática p21, sugerindo o potencial carcinogênico desse 
tipo de poluente ${ }^{4}$.

Ainda em relação aos problemas de pele levantados por essa revisão, os trabalhos de Vierkötter, et $\mathrm{al}^{21}$ e $\mathrm{Li}$, et $\mathrm{al}^{1}$ relataram associação positiva entre a exposição ao material particulado, incluindo compostos orgânicos voláteis, provenientes da queima de combustíveis, com a ocorrência de envelhecimento cutâneo, caracterizado pela presença de rugas grossas e pigmentação distribuída de forma desigual. Esses estudos sugerem que os mecanismos causadores dos efeitos de envelhecimento cutâneo partem da geração de estresse oxidativo. Segundo os pesquisadores, o aumento das espécies reativas de oxigênio, induzido pela ação dos poluentes, acelera os processos de envelhecimento das células da pele por inibir a síntese de colágeno, desregular o sistema de reparo celular de DNA e incrementar a produção de citocinas pró-inflamatórias, acumulando danos que culminam em um aspecto envelhecido da pele.

Com base no exposto, a análise conjunta dos trabalhos avaliados na presente revisão sistemática aponta para um cenário em que o controle ambiental (redução da exposição ao ar contaminado), conforme salienta Kim, et $\mathrm{al}^{17}$, é a principal forma de prevenir a ocorrência de doenças de pele. Contudo, em muitas circunstâncias, é impossível evitar o contato com os poluentes atmosféricos e, nesses casos, a literatura médico científica aponta para uma abordagem terapêutica pautada no emprego de drogas com ação antioxidante e anti-inflamatória, que inibem os efeitos desses contaminantes na saúde da pele humana ${ }^{21}$. Entre os tratamentos mais recentes e eficazes, a presente revisão destaca a dehidroepiandrosterona (DHEA), que protege as células endoteliais de eventos inflamatórios induzidos pelo material particulado ${ }^{22}$ e o éster fenetil do ácido cafeico (CAPE), que possui ação antioxidante ${ }^{23}$.

\section{CONCLUSÕES}

A presente revisão sistemática revelou que poluentes atmosféricos, como os associados ao material particulado e os presentes na fumaça do cigarro causam perturbações na barreira dérmica por meio da produção de estresse oxidativo e citocinas inflamatórias, causando doenças de pele. Entre as doenças mais frequentemente associadas aos contaminantes do ar, segundo a literatura científica avaliada, destacam-se as dermatites atópicas, a alopécia e o envelhecimento cutâneo. A associação entre poluição atmosférica e câncer de pele foi reconhecida em apenas um estudo.

Os resultados dessa revisão também destacam que as crianças representam o grupo mais sensível e investigado quanto às associações entre poluição do ar e doenças de pele, sendo o controle da exposição ambiental a principal forma de prevenção apontada pelos trabalhos revisados. Convém informar que o tratamento das doenças de pele associadas à poluição atmosférica pode ser realizado por meio da utilização de drogas anti-inflamatórias e antioxidantes.

Assim, diante do número reduzido de estudos de abordagem experimental, clínica e epidemiológica, especialmente no Brasil, é importante incentivar as investigações na área da Dermatotoxicologia, especialmente aquelas que priorizem o diagnóstico, a prevenção e o tratamento das doenças de pele associadas aos poluentes atmosféricos.

\section{REFERÊNCIAS}

1. Li M, Vierkötter A, Schikowski T, Hüls A, Ding A, Matsui MS, et al. Epidemiological evidence that indoor air pollution from cooking with solid fuels accelerates skin aging in Chinese women. J Dermatol Sci. 2015 Aug; 79(2):14854. doi: 10.1016/j.jdermsci.2015.04.001. PubMed PMID: 26055797.

2. Brans R, Skudlik C, Weisshaar E, Gediga K, Scheidt R, Wulfhorst B, et al. Association between tobacco smoking and prognosis of occupational hand eczema: a prospective cohort study. Br J Dermatol. 2014 Nov; 171(5):1108-15. doi: 10.1111/bjd.13169. PubMEd PMID: 24909920.

3. Fazzo L, Carere M, Tisano F, Bruno C, Cernigliaro A, Cicero MR, et al. Cancer incidence in Priolo, Sicily: a spatial approach for estimation of industrial air pollution impact. Geospat Health. 2016 Apr; 11(1):320. doi: 10.4081/ gh.2016.320. PubMed PMID: 27087035

4. Kim KE, Cho D, Park HJ. Air pollution and skin diseases: Adverse effects of airborne particulate matter on various skin diseases. Life Sci. 2016 May; 152:126-34. doi: 10.1016/j.Ifs.2016.03.039. PubMed PMID: 27018067.

5. Pereira BB, Cunha PB, Silva GG, Campos EO Jr, Morelli S, Lima EA, et al. Integrated monitoring for environmental health impact assessment related to the genotoxic effects of vehicular pollution in Uberlândia, Brazil. Environ Sci Pollut Res Int. 2017 Jan; 24(3): 2572-2577. doi: 10.1007/s11356-016-8039-5. PubMed PMID: 27826826.

6. Pereira BB, Campos EO Jr, Lima EA, Barrozo MA, Morelli S. Biomonitoring air quality during and after a public transportation strike in the center of Uberlândia, Minas Gerais, Brazil by Tradescantia micronucleus bioassay. Environ Sci Pollut Res Int. 2014 Mar; 21(5):3680-5. doi: 10.1007/s11356-013-2335-0. PubMed PMID: 24277431.

7. Pénard-Morand C, Raherison C, Charpin D, Kopferschmitt C, Lavaud F, Caillaud D, et al. Long-term exposure to close-proximity air pollution and asthma and allergies in urban children. Eur Respir J. $2010 \mathrm{Jul}$; 36(1):33-40. doi: 10.1183/09031936.00116109. PubMed PMID: 20075054.

8. Lademann J, Schaefer H, Otberg N, Teichmann A, Blume-Peytavi U, Sterry W. Penetration of microparticles into human skin. Hautarzt. 2004 Dec; 55(12):11171119. doi: 10.1007/s00105-004-0841-1. PubMed PMID: 15568130.

9. Moher D, Liberati A, Tetzlaff J, Altman DG. The Prisma Group. Preferred Reporting Items For Systematic Reviews And Meta-Analyses: the PRISMA Statement. PLoS Med. 2009 Jul; 6(7):e1000097. doi: 10.1371/journal. pmed.1000097. PubMed PMID: 19621072.

10. Kwon JH, Kim E, Chang MH, Park EA, Hong YC, Ha M, et al. Indoor total volatile organic compounds exposure at 6 months followed by atopic dermatitis at 3 years in children. Pediatr Allergy Immunol. 2015 Jun; 26(4):352-8. doi: 10.1111/pai.12393.

11. Lee YL, Li CW, Sung FC, Guo YL. Increasing prevalence of atopic eczema in Taiwanese adolescents from 1995 to 2001. Clin Exp Allergy. 2007 Apr; 37(4):543- 


\section{1. doi: 10.1111/j.1365-2222.2007.02690.x. PubMed PMID: 17430051.}

12. Kallawicha K, Chuang YC, Lung SC, Han BC, Ting YF, Chao HJ. Exposure to ambient bioaerosols is associated with allergic skin diseases in Greater Taipei residents. Environ Pollut. 2016 Sept; 216:845-50. doi: 10.1016/j. envpol.2016.06.057.

13. Solé D, Camelo-Nunes IC, Wandalsen GF, Pastorino AC, Jacob CM, Gonzalez C, et al. Prevalence of symptoms of asthma, rhinitis, and atopic eczema in Brazilian adolescents related to exposure to gaseous air pollutants and socioeconomic status. J Investig Allergol Clin Immunol. 2007; 17(1):6-13. PubMed PMID: 17323857.

14. Amancio CT, Nascimento LFC. Asma e poluentes ambientais: um estudo de séries temporais. Rev Assoc Med Bras. 2012 Maio-Jun; 58(3): 302-307. http:// dx.doi.org/10.1590/S0104-42302012000300009.

15. Gatherwright J, Liu MT, Gliniak C, Totonchi A, Guyuron B. The contribution of endogenous and exogenous factors to female alopecia: a study of identical twins. Plast Reconstr Surg. 2012 Dec; 130(6):1219-1226. doi: 10.1097/ PRS.0b013e31826d104f. PubMed PMID: 22878477.

16. Gatherwright J, Liu MT, Amirlak B, Gliniak C, Totonchi A, Guyuron B. The contribution of endogenous and exogenous factors to male alopecia: a study of identical twins. Plast Reconstr Surg. 2013 May; 131(5):794e-801e. doi: 10.1097/ PRS.0b013e3182865ca9. PubMed PMID: 23629119.

17. Kim HO, Kim JH, Cho SI, Chung BY, Ahn IS, Lee $\mathrm{CH}$, et al. Improvement of atopic dermatitis severity after reducing indoor air pollutants. Ann Dermatol. 2013 Aug; 25(3): 292-297. doi: 10.5021/ad.2013.25.3.292.

18. Kim J, Kim EH, Oh I, Jung K, Han Y, Cheong HK, et al. Symptoms of atopic dermatitis are influenced by outdoor air pollution. J Allergy Clin Immunol. 2013 Aug; 132(2):495-8.e1. doi: 10.1016/j.jaci.2013.04.019. PubMed PMID: 23763977.

19. Kathuria P, Silverberg JI. Association of pollution and climate with atopic eczema in US children. Pediatr Allergy Immunol. 2016 Aug; 27(5):478-85. doi: 10.1111/pai.12543. PubMed PMID: 26842875.

20. Su LH, Chen TH. Association of androgenetic alopecia with smoking and its prevalence among Asian men: a community-based survey. Arch Dermatol. 2007; 143(11):1401-6. doi: 10.1001/archderm.143.11.1401. PubMed PMID: 18025364.

21. Vierkötter A, Schikowski T, Ranft U, Sugiri D, Matsui $M$, Krämer $U$, et al. Airborne particle exposure and extrinsic skin aging. J Invest Dermatol. $2010 \mathrm{Dec}$; 130(12): 2719-26. doi: 10.1038/jid.2010.204. PubMed PMID: 20664556.

22. Huerta-Garcia E, Montiel-Davalos A, Alfaro-Moreno E, Gutierrez-Iglesias G, Lopez-Marure R. Dehydroepiandrosterone protects endothelial cells against inflamatory events induced by urban particulate matter and titanium dioxide nanoparticles. Biomed Res Int. 2013; 2013:382058. doi: 10.1155/2013/382058. PubMed PMID: 23484113

23. Jo SY, Lee N, Hong SM, Jung HH, Chae SW. Caffeic acid phenethyl ester inhibits diesel exhaust particle-induced inflammation of human middle ear epithelial cells via NOX4 inhibition. Ann Otol Rhinol Laryngol. 2013 Sep; 122(9): 595-600. doi: 10.1177/000348941312200910.

24. Altuğ H, Gaga EO, Döğeroğlu T, Ozden O, Ornektekin S, Brunekreef B, et al. Effects of air pollution on lung function and symptoms of asthma, rhinitis and eczema in primary school children. Environ Sci Pollut Res Int. 2013 Sep; 20(9): 6455-67. doi: 10.1007/s11356-013-1674-1. PubMed PMID: 23591930.

25. Graif $Y$, German L, Ifrah A, Livne I, Shohat T. Dose-response association between smoking and atopic eczema: results from a large cross-sectional study in adolescents. Dermatology. 2013; 226(3):195-9. doi: 10.1159/000348333. PubMed PMID: 23711459.
26. Herbarth O, Fritz GJ, Rehwagen M, Richter M, Röder S, Schlink U. Association between indoor renovation activities and eczema in early childhood. Int J Hyg Environ Health. 2006 May; 209(3):241-7. doi: 10.1016/j.ijheh.2006.01.003.

27. Huang CC, Wen HJ, Chen PC, Chiang TL, Lin SJ, Guo YL. Prenatal air pollutant exposure and occurrence of atopic dermatitis. Br J Dermatol. 2015 Oct; 173(4):981-8. doi: 10.1111/bjd.14039. PubMed PMID: 26202732.

28. Jedrychowski W, Perera F, Maugeri U, Mrozek-Budzyn D, Miller RL, Flak E, et al. Effects of Prenatal and Perinatal Exposure to Fine Air Pollutants and Maternal Fish Consumption on the Occurrence of Infantile Eczema. Int Arch Allergy Immunol. 2011; 155(3):275-81. doi: 10.1159/000320376. PubMed PMID: 21293147.

29. Kim E-H, Kim S, Lee JH, Kim J, Han Y, Kim Y-M, et al. Indoor Air Pollution Aggravates Symptoms of Atopic Dermatitis in Children. PLoS One. 2015 Mar; 10(3):e0119501. doi: 10.1371/journal.pone.0119501. PubMed PMID: 25781186

30. Krämer U, Sugiri D, Ranft U, Krutmann J, Von Berg A, Berdel D, Behrendt H, et al. Eczema, respiratory allergies, and traffic-related air pollution in birth cohorts from small-town areas. J Dermatol Sci. 2009 Nov; 56(2):99-105. doi: 10.1016/j. jdermsci.2009.07.014. PubMed PMID: 19713084.

31. Lee $\mathrm{CH}$, Chuang HY, Hong $\mathrm{CH}$, Huang SK, Chang YC, Ko YC, et al. Lifetime exposure to cigarette smoking and the development of adult-onset atopic dermatitis. Br J Dermatol. 2011 Mar; 164(3): 483-9. doi: 10.1111/j.13652133.2010.10116.x.

32. Lee JH, Suh J, Kim EH, Cho JB, Park HY, Kim J, et al. Surveillance of home environment in children with atopic dermatitis: a questionnaire survey. Asia Pac Allergy. 2012 Jan; 2(1):59-66. doi: 10.5415/apallergy.2012.2.1.59. PubMed PMID: 22348208.

33. Lee JH, Lee HS, Park MR, Lee SW, Kim EH, Cho JB, et al. Relationship between indoor air pollutant levels and residential environment in children with atopic dermatitis. Allergy Asthma Immunol Res. 2014 Nov; 6(6): 517-24. doi: 10.4168/ aair.2014.6.6.517. PubMed PMID: 25374751.

34. Lee YL, Su HJ, Sheu HM, Yu HS, Guo YL. Traffic-related air pollution, climate, and prevalence of eczema in Taiwanese school children. J Invest Dermatol. 2008 Oct; 128(10): 2412-20. doi: 10.1038/jid.2008.110. PubMed PMID: 18449213.

35. Morgenstern V, Zutavern A, Cyrys J, Brockow I, Koletzko S, Krämer U, et al. Atopic diseases, allergic sensitization, and exposure to traffic-related air pollution in children. Am J Respir Crit Care Med. 2008 Jun; 177(12): 1331-7. doi: 10.1164/rccm.200701-0360C. PubMed PMID: 18337595.

36. Schmitz R, Atzpodien K, Schlaud M. Prevalence and risk factors of atopic diseases in German children and adolescents. Pediatr Allergy Immunol. 2012 Dec; 23(8):716-23. doi: 10.1111/j.1399-3038.2012.01342.x. PubMed PMID: 22882467.

37. Seo S, Kim D, Min S, Paul C, Yoo Y, Choung JT. GIS-based Association Between PM10 and Allergic Diseases in Seoul: Implications for Health and Environmental Policy. Allergy Asthma Immunol Res. 2016 Jan; 8(1): 32-40. doi: 10.4168/ aair.2016.8.1.32

38. Song S, Lee K, Lee YM, Lee JH, Lee SI, Yu SD, et al. Acute health effects of urban fine and ultrafine particles on children with atopic dermatitis. Environ Res. 2011 Apr; 111(3): 394-9. doi: 10.1016/j.envres.2010.10.010. PubMed PMID: 21367405.

39. Wang IJ, Hsieh WS, Wu KY, Guo YL, Hwang YH, Jee SH, et al. Effect of gestational smoke exposure on atopic dermatitis in the offspring. Pediatr Allergy Immunol. 2008 Nov; 19(7): 580-6. doi: 10.1111/j.1399-3038.2008.00759.x. PubMed PMID: 18540992.

40. Wang IJ, Guo YL, Lin TJ, Chen PC, Wu YN. GSTM1, GSTP1, prenatal smoke 
exposure, and atopic dermatitis. Ann Allergy Asthma Immunol. 2010 Aug; 105(2): 124-9. doi: 10.1016/j.anai.2010.04.017. PubMed PMID: 20674822

41. Wang IJ, Chen SL, Lu TP, Chuang EY, Chen PC. Prenatal smoke exposure, DNA methylation, and childhood atopic dermatitis. Clin Exp Allergy. 2013; 43(5):535-
43. doi: 10.1111/cea.12108. PubMed PMID: 23600544.

42. Yi O, Kwon HJ, Kim H, Ha M, Hong SJ, Hong YC, et al. Effect of environmental tobacco smoke on atopic dermatitis among children in Korea. Environ Res. 2012 Feb; 113: 40-5. doi: 10.1016/j.envres.2011.12.012. PubMed PMID: 22264877. 